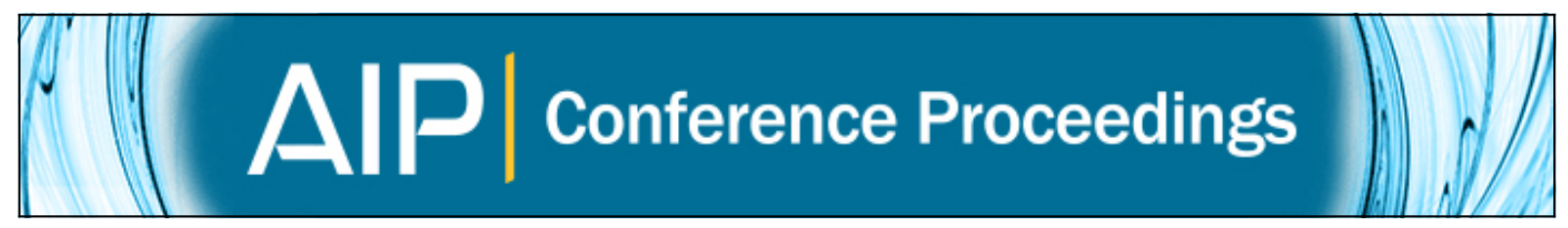

\title{
Shot-noise at a Fermi-edge singularity: Non-Markovian dynamics
}

N. Ubbelohde, K. Roszak, F. Hohls, N. Maire, T. Novotný, and R. J. Haug

Citation: AIP Conference Proceedings 1566, 225 (2013); doi: 10.1063/1.4848367

View online: http://dx.doi.org/10.1063/1.4848367

View Table of Contents: http://scitation.aip.org/content/aip/proceeding/aipcp/1566?ver=pdfcov

Published by the AIP Publishing

\section{Articles you may be interested in}

Non-Markovian shot noise spectrum of quantum transport through quantum dots

J. Appl. Phys. 109, 053704 (2011); 10.1063/1.3555586

Control of the non-Markovian dynamics of a qubit

Appl. Phys. Lett. 89, 254102 (2006); 10.1063/1.2403923

Non-Markovian stochastic processes: Colored noise

Chaos 15, 026107 (2005); 10.1063/1.1860471

Rate processes with non-Markovian dynamical disorder

J. Chem. Phys. 122, 164506 (2005); 10.1063/1.1884087

The generalized Smoluchowski equation and non-Markovian dynamics

J. Chem. Phys. 84, 5824 (1986); 10.1063/1.449892 


\title{
Shot-Noise at a Fermi-Edge Singularity: Non-Markovian Dynamics
}

\author{
N. Ubbelohde*, K. Roszak ${ }^{\dagger}$, F. Hohls**, N. Maire*, T. Novotný ${ }^{\ddagger}$ and R. J. Haug* \\ *Institut für Festkörperphysik, Leibniz Universität Hannover, Appelstraße 2, D-30167 Hannover, Germany \\ ${ }^{\dagger}$ Institute of Physics, Wroctaw University of Technology, PL-50370 Wroctaw, Poland \\ **Physikalisch-Technische Bundesanstalt, D-38116 Braunschweig, Germany \\ ${ }^{\ddagger}$ Department of Condensed Matter Physics, Faculty of Mathematics and Physics, Charles University, CZ-12116 \\ Prague, Czech Republic
}

\begin{abstract}
.
For an InAs quantum dot we study the current shot noise at a Fermi-edge singularity in low temperature cross-correlation measurements. In the regime of the interaction effect the strong suppression of noise observed at zero magnetic field and the sequence of enhancement and suppression in magnetic field go beyond a Markovian master equation model. Qualitative and quantitative agreement can however be achieved by a generalized master equation model taking non-Markovian dynamics
\end{abstract} into account.

Keywords: electron transport, shot noise, non-Markovian dynamics, Fermi-edge singularity

PACS: $72.70 .+\mathrm{m}, 72.10 . \mathrm{Fk}, 73.63 . \mathrm{Kv}, 73.23 . \mathrm{Hk}$

In resonant tunneling through InAs quantum dots the Fermi-edge singularity (FES) is observed as a strong overshoot of the current at the resonance of a quantum dot level and the Fermi-edge of the lead caused by an enhanced tunneling probability $[1,2,3]$. While the dynamics of single electron transport due to the Coulomb blockade mechanism are well described by a rate equation model [4], there exist significant discrepancies in the regime of the Fermi-edge singularity [5]. In measurements of the electron shot noise at low temperatures these deviations can be identified as an effect of quantum correlations between dot and fermionic reservoir giving rise to non-Markovian corrections in a generalized master equation model $[6,7,8]$.

The sample structure consists of self assembled InAs quantum dots embedded between AlAs barriers in a GaAs-AlAs-GaAs resonant tunneling device. The (zerofrequency) shot noise power $S$ is measured in a dilution refrigerator at temperatures down to $70 \mathrm{mK}$ using two cryogenic amplifiers in a cross-correlation configuration [8]. The Fano factor F quantifies the shot noise in a comparison with the expected noise of an ideal Poissonian noise source $F=S / 2 e I$.

Figure 1 shows the current-voltage characteristics and Fano factor. On resonance of the quantum dot ground state with the Fermi energy of the emitter the current displays the sharp peak and power law decline of the FES. For further increased bias (V $>170 \mathrm{mV}$ ) the current continues to decrease due to the three dimensional density of states (DOS) in the lead. In this regime current and Fano factor can be described by a Markovian master equation

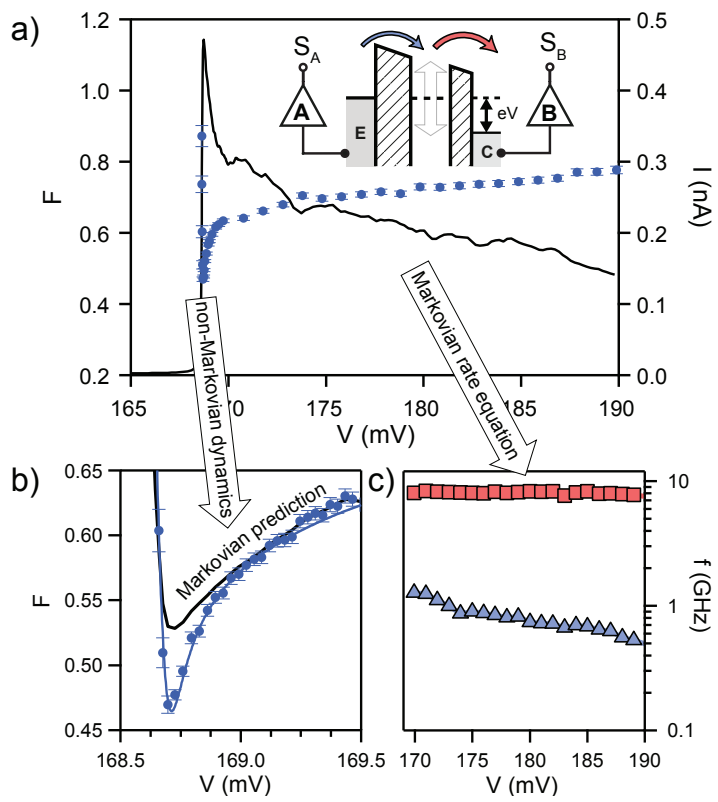

FIGURE 1. (a) Current (line) and Fano factor (symbols) depending on the bias voltage. For large bias the tunneling rates can be extracted from a Markovian master equation model (c). In the regime of the FES (b) the Markovian model clearly deviates from measured Fano factor.

model as functions of the emitter and collector tunneling rates. The deduced tunneling rates shown in Fig. 1.c reflect the asymmetry of the AlAs barriers of the sample 


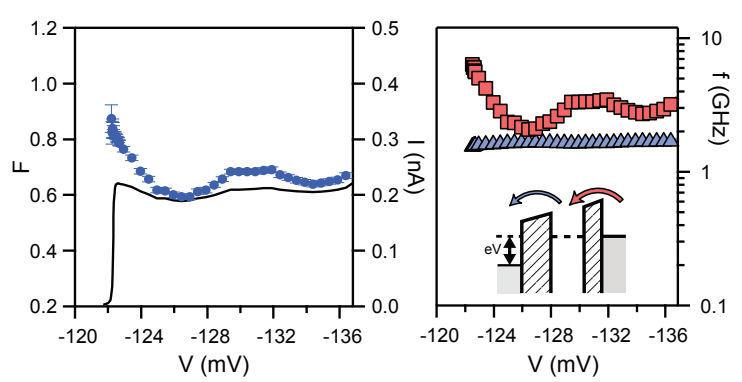

FIGURE 2. Left: Current (line) and Fano factor (symbols) under reversed bias without the influence of the interaction effect. Right: Tunneling rates determined with a Markovian master equation model.

structure. The collector rate is nearly constant and the emitter rate follows the energy dependence of the emitter DOS.

Assuming the collector rate to be constant allows for the extrapolation of the emitter tunneling rate from the average current alone. For these extrapolated tunneling rates the Markovian master equation model predicts a Fano factor, which clearly deviates from the measured value in Fig. 1.b. In comparison, under reversed bias the sample does not show a current overshoot at the Fermiedge (Fig. 2) and the measured noise is well modeled by the Markovian master equation up to the Fermi-edge, where back-tunneling into the emitter starts to play a role. The two tunneling rates following from this model correspond to the now reversed symmetry of the barriers. Again we observe no variations for the tunneling rate into the collecting lead, while the modulation of the tunneling rate corresponding to the emitting lead is likely to stem from fluctuations in the DOS $[9,10]$.

The additional correlations introduced by the many particle interaction effect therefore require a new theoretical description beyond the Markovian model used so far. A non-Markovian generalized master equation model [8] allows for a more elaborate analysis of the experimental data for various values of temperature and magnetic field. Additional correlations due to bidirectional tunneling coupling to the emitter and the mutual Coulomb blockade between Zeeman split dot levels are also taken into account.

In the comparison of theory and experiment we are not only considering the spin-degenerate FES but also the spin-split case in magnetic field. With a field applied parallel to the current we observe a independent FES for each Zeeman level. The model parameters can be determined by fitting the mean current at different temperatures using one set of parameters [8]. Fig. 3 compares the resulting Fano factor with the experiment. The effect of the FES is again observed as a strong reduction of the

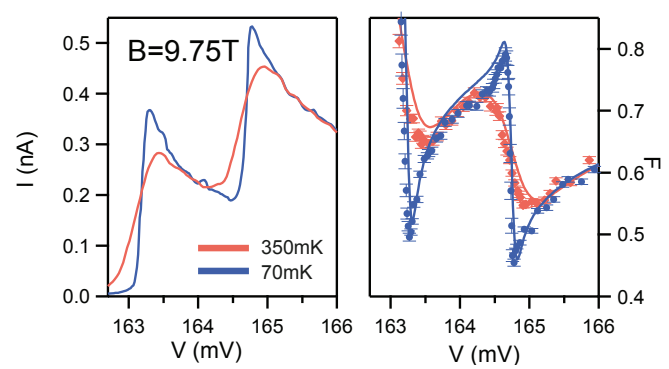

FIGURE 3. Current (left) and a comparison of the Fano factor (right) calculated with the non-Markovian model (lines) and the measurement (symbols) in magnetic field $(B=9.75 \mathrm{~T})$. Two selected temperatures illustrate the strong temperature dependence of the non-Markovian corrections.

Fano factor which quantitatively cannot be explained by an interaction induced enhancement of the emitter tunneling rate. At the onset of the second Zeeman level we furthermore observe a qualitative different behavior, as the Fano factor is first enhanced and then suppressed. These correlation effects show a strong temperature dependence as the enhancement of the Fano factor vanishes for even a slightly elevated temperature of $350 \mathrm{mK}$.

The theoretical prediction of the Fano factor, represented by the solid lines in Fig. 3 (blue line in Fig. 1.b), correspond remarkably with above observations and the qualitative and quantitative agreement illustrates the importance of the non-Markovian corrections.

This work was supported by the German Excellence Initiative via QUEST (Hannover), by the Czech Science Foundation via Grant No. 204/11/J042 (Prague), and the TEAM programme of the Foundation for Polish Science, co-financed from the European Regional Development Fund (K.R.),

\section{REFERENCES}

1. K. A. Matveev, and A. I. Larkin, Phys. Rev. B 46, 15337 (1992).

2. A. K. Geim, et al., Phys. Rev. Lett. 72, 2061-2064 (1994).

3. I. Hapke-Wurst, et al., Phys. Rev. B 62, 12621 (2000).

4. A. Nauen, et al., Phys. Rev. B 70, 033305 (2004).

5. N. Maire, et al., Phys. Rev. B 75, 233304 (2007).

6. C. Flindt, et al., Phys. Rev. Lett. 100, 150601 (2008).

7. C. Flindt, et al., Phys. Rev. B 82, 155407 (2010).

8. N. Ubbelohde, et al., Sci. Rep. 2,374 (2012).

9. T. Schmidt, et al., EPL (Europhysics Letters) 36, 61 (1996).

10. T. Schmidt, et al., Phys. Rev. Lett. 86, 276-279 (2001). 